\title{
COMPUTING THE FIXED-POINTS OF GENERAL MIXED VARIATIONAL INEQUALITIES
}

\author{
MUHAMMAD ASLAM NOOR
}

\begin{abstract}
In this paper, we suggest and analyze a class of predictor-corrector methods for computing the fixed-points of general mixed variational inequalities. The convergence of the proposed methods only requires the partially relaxed strongly monotonicity of the operator, which is weaker than co-coercivity. As special cases, we obtain a number of known and new results for solving various classes of variational inequalities and related problems.
\end{abstract}

Mathematics subject classification (2000): 49J40, 90C33.

Key words and phrases: Variational inequalities, auxiliary principle, iterative methods, convergence, fixed points.

\section{REFERENCES}

[1] A. S. ANTIPIN, Iterative gradient prediction-type methods for computing fixed points of extremal mapping, Parametric Optimization and Related Topics IV. (eds. J. Guddat, H. Th. Jongen, F. Nizicka, G. Still and F. Twitt), Peter Lang, Frankfurt am Main, (1997), 11-24.

[2] R. W. COTtLe, F. GiAnNeSSI AND J. L. LiOns, Variational Inequalities and Complementarity Problems: Theory and Applications, J. Wiley and Sons, New York, 1980.

[3] F. GianNeSSI AND A. MAUGERI, Variational Inequalities and Network Equilibrium Problems, Plenum Press, New York, 1995.

[4] R. Glowinski, J. L. Lions ANd R. Trémolières, Numerical Analysis of Variational Inequalities, North-Holland, Amsterdam, 1981.

[5] J. L. Lions And G. StampacchiA, Variational inequalities, Comm. Pure Appl. Math. 20 (1967), $493-512$.

[6] Z. Naniewicz and P. D. PANagiotopoulos, Mathematical Theory of Hemivariational Inequalities and Applications, Marcel Dekker, New York, 1995.

[7] M. Aslam NoOR, A class of new iterative methods for general mixed variational inequalities, Math. Computer Modelling, 31 (2000), 11-19.

[8] M. AsLam Noor, Algorithms for general monotone mixed variational inequalities, J. Math. Anal. Appl. 229 (1999), 330-343.

[9] M. Aslam Noor, General variational inequalities, Appl. Math. Letters 1 (1988), 119-121.

[10] M. Aslam NooR, Wiener-Hopf equations and variational inequalities, J. Optim. Theory Appl. 79 (1993), 197-206.

[11] M. AsLam Noor, An extraresolvent method for monotone mixed variational inequalities, Math. Computer Modelling 29 (1999), 95-100.

[12] M. ASlam NoOR, Some recent advances in variational inequalities, Part I, basic concepts, New Zealand J. Math. 26 (1997), 53-80.

[13] M. Aslam Noor, Some recent advances in variational inequalities, Part II, other concepts, New Zealand J. Math. 26 (1997), 229-255.

[14] M. ASLAm NOOR, Some algorithms for general monotone mixed variational inequalities, Math. Computer Modelling 29 (7) (1999), 1-9.

[15] M. Aslam NooR, A new predictor-corrector method for noncoercive mixed variational inequalities, Korean J. Comput. Appl. Math. 7 (2) (2000), 363-371. 
[16] G. Stampacchia, Formes bilineaires coercivities sur les ensembles convexes, C. R. Acad. Sci. Paris, 258 (1964), 4413-4416.

[17] P. TSENG, A modified forward-backward splitting method for maximal monotone mappings, SIAM J. Control Optim. 38 (2000), 431-446.

[18] D. L. ZhU AND P. MARCOTTE, An extended descent framework for variational inequalities, J. Optim. Theory Appl. 80 (1994), 349-366. 